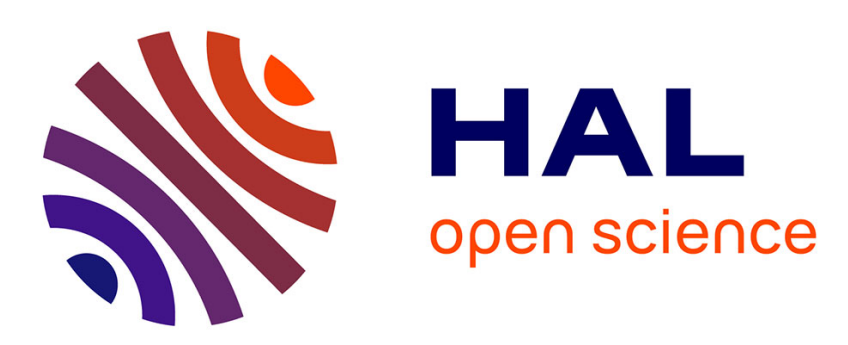

\title{
Isogonal moulding surfaces: A family of shapes for high node congruence in free-form structures
}

Romain Mesnil, Cyril Douthe, Olivier Baverel, Bruno Léger, Jean-François

Caron

\section{- To cite this version:}

Romain Mesnil, Cyril Douthe, Olivier Baverel, Bruno Léger, Jean-François Caron. Isogonal moulding surfaces: A family of shapes for high node congruence in free-form structures. Automation in Construction, 2015, 59, pp.38-47. 10.1016/j.autcon.2015.07.009 . hal-01200525

\section{HAL Id: hal-01200525 \\ https://hal.science/hal-01200525}

Submitted on 16 Sep 2015

HAL is a multi-disciplinary open access archive for the deposit and dissemination of scientific research documents, whether they are published or not. The documents may come from teaching and research institutions in France or abroad, or from public or private research centers.
L'archive ouverte pluridisciplinaire HAL, est destinée au dépôt et à la diffusion de documents scientifiques de niveau recherche, publiés ou non, émanant des établissements d'enseignement et de recherche français ou étrangers, des laboratoires publics ou privés. 


\title{
Isogonal moulding surfaces: a family of shapes for high node congruence in free-form structures
}

\author{
Romain Mesnil $^{\mathrm{a}, \mathrm{c}, *}$, Cyril Douthe ${ }^{\mathrm{b}}$, Olivier Baverel ${ }^{\mathrm{a}}$, Bruno Léger ${ }^{\mathrm{c}}$, Jean-François Caron $^{\mathrm{a}}$ \\ ${ }^{a}$ Université Paris-Est, Laboratoire Navier (UMR 8205), ENPC, IFSTTAR, CNRS, Marne-La-Vallée, France \\ ${ }^{b}$ Université Paris-Est, IFSTTAR, France \\ ${ }^{c}$ Bouygues Construction SA, Guyancourt, France
}

\begin{abstract}
The design of free-form structures is governed by structural and geometric considerations, the latter ones being closely linked to the costs of fabrication. If some construction constraints have been studied extensively, the question of the repeatability of nodes in free-form structures has rarely been addressed yet. In this paper, a family of surfaces that can be optimized regarding typical geometrical constraints and that exhibit high node congruence is proposed. They correspond to particular meshes of moulding surfaces and are called isogonal moulding surfaces by the authors. The geometrical properties of these surfaces are discussed. In particular, it is shown how to derive Edge Offset Mesh from them. It is also demonstrated that they represent all the possible meshes parallel to surfaces of revolution. Finally, the reader is introduced to some computational strategies linked to isogonal moulding surfaces.
\end{abstract}

Keywords: Free-form architecture, parametric design, fabrication-aware design, gridshell, structural morphology

\section{Introduction}

Complex shapes play an increasing role in modern architecture. The development of both NURBS modeling and computer aided industrial processes has allowed new formal possibilities. The term of free-form usually describes these new shapes that have emerged. The creation of formal freedom is however limited by physical constraints linked to structural performance and fabrication. One of the designers' concerns is indeed to assure the ease of manufacturing of the different elements, leading to what can be called geometric optimization. Two design philosophies are opposing. The top-down approach, which considers a given shape and tries to optimize its discrete counterpart, has been given a lot of attention recently [11]. The bottom-up approach uses a concatenation of simple shapes that have desirable properties. It offers a great control over the properties of both structural layout and facade from early stage, the drawback being that its formal possibilities are restricted (see for example [19]). In this paper, new tools for a bottom-up approach are provided.

The paper is organized as follows. Section 2 discusses the different design methodologies for doubly curved systems. It also sums up the existing strategies for the geometric optimization of free-form structures. Among typical criteria of optimization, a new one based on the repeatability of nodes is proposed. A new family of surfaces that have high node congruence, called isogonal moulding

\footnotetext{
* Corresponding author

E-mail: romain.mesnil@enpc.fr
}

surfaces, is introduced in Section 3. It is shown that these surfaces admit Edge Offset Meshes, which are optimal in terms of manufacturing. Numerical strategies related to these shapes are proposed in Section 4. A conclusion finally discusses the potential of these structures in Section 5 .

\section{Geometric optimization of free-form structures}

\subsection{Geometric optimization objectives}

The geometrical optimization aims at controlling the properties of a discrete system. These properties can be linked with the repeatability of panels and structural components or to their intrinsic properties. The usual optimization objectives are the following:

- Planarity of panels: Cutting a panel from a flat sheet is indeed significantly less expensive than creating a complex doubly curved mould. This constraint was integrated from the very beginning of free-form architecture [17]. Recent realizations using cold bent glass show that singly curved panels are a good alternative to planar panels [2]. A more detailed discussion on this topic is proposed in [16]. The link between planar quadrilateral meshes and the developable surfaces is direct, since a developable surface can be defined as the envelope of a family of planes.

- Torsion-free nodes and Conical Mesh: A torsion-free node is a node where the central plane of 
beams are all co-axial, as shown in Figure 1. From a constructive point of view, these nodes can be given an axis and are easier to produce. Moreover, they are compatible with double layer grids [11]. A mesh with torsion-free nodes is also called a Conical Mesh, because all the faces belonging to the same node are tangent to the same cone.

- Perfect nodes and Edge Offset Mesh: Even for torsion-free layouts, the alignment of beams in three dimensions is a sensitive topic. If one builds with constant height elements (which is often the case for steel gridshells), then only few meshes guarantee that both the top and the bottom of the members will perfectly match at any vertex. Such meshes are known as Edge Offset Meshes, their nodes called perfect nodes and constitute a subset of Conical Meshes. They have remarkable mathematical properties described in [14].

- Uniform member length: This criterion is linked to constraints on the maximal width of panels (minimization of material loss through cutting and maximal statically allowable span), but also to aesthetic considerations. This optimization goal is less sensitive to cost than the first ones. Nonetheless, some buildings used a uniform member length to map a doubly curved surface: this was the case of a circular dome over a swimming pool in Neckarsulm [18], and this is also a key characteristic of elastic gridshells [1]. Different tools, including the compass method used by the Institute for Lightweight Structures of Stuttgart [13], have been developed for this purpose.

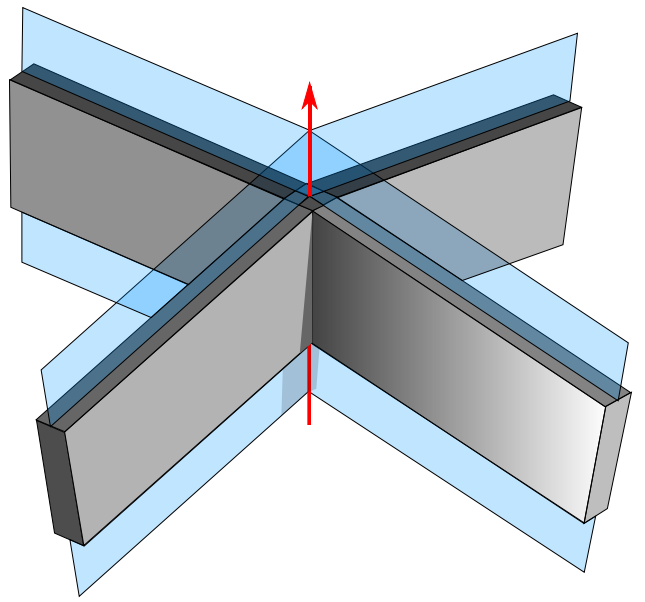

Figure 1: A torsion-free node, where central planes meet at the same axis (arrow).

These design constraints can be solved by different strategies. A common solution to deal with free-form architecture is to use triangular panels. They are indeed always planar, but their high valence implies heavy and complex nodes. Moreover, no torsion-free layout for triangular meshes exists apart from very specific cases. For these reasons, translation surfaces were introduced in the 1990's [17]. They guarantee planar quadrilateral panels, leading to better connection details, less mullions and less loss of material through cutting. The formal possibilities offered by surfaces covered with planar quads have been broaden with the introduction of scale-trans-surfaces [7]. However, the nodes of translation surfaces are generally not torsion-free, which sets limits to the maximal span of these structures, since only single layer structures are possible.

The development of discrete differential geometry and computer aided design allowed a better understanding of the mathematical properties of Planar Quadrilateral (PQ) meshes. In particular, the interest of using a network of curvature lines has been explained in [11]: these networks are indeed very close to PQ conical meshes and can be computed on any smooth surfaces. It has also been shown that properties of meshes with torsion-free nodes and planar panels can be written with simple formulæ on angles or lengths [22]. Methods of energy minimization have therefore been used in order to approximate conical, circular or PQ-meshes. These methods allow the manipulation of meshes under geometrical constraints thanks to real-time optimization [23, 3].

A simple assessment of these methods for geometrical generation and optimization of form is proposed in Table 1 . One + sign means that only a partial answer to the problem is provided. The ++ signs mean that the problem is completely treated by the strategy offered. A - sign shows that a solution performs poorly for a given optimisation goal. The -- sign indicates that a strategy is not suited for the considered optimisation task, and should be used with consideration. Even though only geometrical constraints are presented, it can be noticed that no method gives a fully satisfactory answer to all the optimisation objectives. This illustrates the problem that designers have to face when starting a project: the choice of the hierarchy between geometrical optimization targets requires indeed a thorough knowledge of relative costs of panels, connections and members.

Finally, it is noticed that conical PQ-meshes are highly efficient for factors which are the most sensitive to cost. Edge Offset Meshes are also of interest, but all their properties are not fully known yet. The link between Edge Offset Meshes and Koebe polyhedra has been discussed in [15], where answers on the design of Edge Offset Meshes with hexagons are given. The question of the design freedom offered by Edge Offset Quadrilateral Meshes is still open to this day. It was indeed stated in [14] that "EdgeOffset Meshes are discrete versions of Laguerre isothermic surfaces [...]. Especially for applications in architectural design it would be highly interesting to get more insight of the possible shapes of Laguerre isothermic surfaces.". The design possibilities offered by isothermic surfaces are however broader than the one simply offered by surfaces of 


\begin{tabular}{|c|c|c|c|c|c|}
\hline Optimisation & $\begin{array}{c}\text { Angles } \\
\text { repetition }\end{array}$ & $\begin{array}{c}\text { Length } \\
\text { uniformity }\end{array}$ & $\begin{array}{c}\text { Panels } \\
\text { planarity }\end{array}$ & $\begin{array}{c}\text { Node } \\
\text { complexity }\end{array}$ & $\begin{array}{c}\text { Design } \\
\text { freedom }\end{array}$ \\
\hline Triangular Mesh & - & ++ & ++ & -- & ++ \\
\hline Scale-trans surfaces & -- & ++ & ++ & - & -- \\
\hline PQ Conical Mesh & + & + & ++ & + & + \\
\hline Edge Offset Mesh & + & - & ++ & ++ & - \\
\hline
\end{tabular}

Table 1: Assessement of different methods of geometrical optimisation.

translation.

\subsection{New optimization goal and problem statement}

The question of the regularity of connection details seems to be of little concern. The main reason for this is that automatic design processes manage the uniqueness of each connection $[10,20]$. However, this constrains the choice of the technologies used at early stages in the design. Moreover, no scale effect can be used to save costs on connections, which remains an important factor on the economy of steel gridshells. This problem has been identified early in the design of free-form, and some structural systems were proposed in order to deal directly with this constraint. The most known is the elastic gridshell: the structure is built from an initially flat grid with no initial in-plane shear stiffness which is deformed in order to map a given shape [13]. This procedure has been used for one of the most significant designs of free-form of the modern era: the Multihalle in Mannheim [9]. One unique connection detail is used in order to map the free-form shape found by inversion of an hanging net. This fact has guaranteed a reasonable cost for these structures. Recent developments in this field have broadened the possibilities of elastic gridshells with the introduction of composite materials, such as GFRP $[6,1]$. However, these structures feature very slender elements and have therefore a limited span. They also do not consider the geometry of the covering panels, which have to be optimized separately.

The question of structures that would be optimal for the classical optimization goals and with a high node congruence is thus largely unexplored. The profusion and the mathematical sophistication of the presented optimization goals makes it also difficult for non-specialists to chose a design strategy at early stages. In addition, no simple way to generate good shapes (for example, the ones that yield to quadrilateral Edge Offset Meshes) is given apart from scale-trans surfaces and simple surfaces, such as developable surfaces or surfaces of revolution. This leads to the two questions that are addressed in this article:

- Is there a family of intrinsically simple shapes that can be optimal for all the geometrical criteria exposed above, and especially for the repeatability of connections?

- Is there a simple and intuitive way to generate quadrilateral Edge Offset Meshes and other optimal meshes?

\section{Isogonal moulding surfaces}

It will be shown that isogonal moulding surfaces offer a positive answer to these questions. These surfaces are indeed specific cases of Monge's surfaces which define a large family of forms which can be easily and naturally meshed by PQ conical meshes. The intrinsic properties of isogonal moulding surfaces are discussed in Section 3.4, in particular, it is shown that they can be optimized towards Edge Offset Meshes.

\subsection{Monge's surfaces}

Monge's surfaces (also known as generalised moulding surface) are a family of surfaces introduced by the French mathematician Gaspard Monge at the beginning of the nineteenth century in his lectures on differential geometry [12]. Several equivalent definitions of these surfaces are given in lectures and are recalled in [15]. The generation of Monge's surfaces can be described by a kinematic procedure, represented on Figure 2. The surface is generated by the sweeping of a planar curve, called generatrix (in red on the Figure) along another curve, called rail-curve (in blue). If the rail-curve is planar, then the surface is called a moulding surface. There are some restriction on the kinematic of the generatix, since it has to lay in the normal plane of the rail curve and it has to follow a rotation minimizing frame. Monge's surfaces are therefore a specific case of sweeping surfaces, which makes them a very familiar set of surfaces for designers.

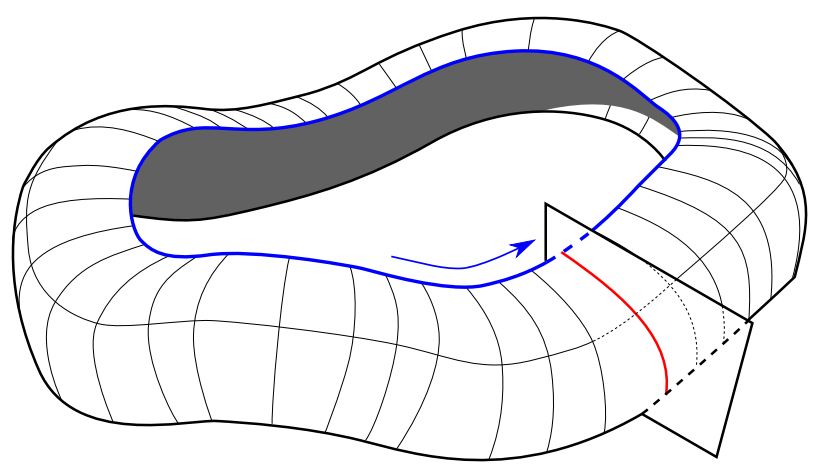

Figure 2: Kinematic shape generation of Monge's surface: generatrix (red) and parallel (blue).

The iso-curves of the surface are called respectively generatrices and parallels. Generatrices and parallels are 
the curvature lines of Monge's surfaces, and therefore form a conjugate-curves network. A discrete version of these lines is therefore close to a conical PQ-mesh [11]. Monge also demonstrated [12] that generatrices are geodesics of Monge's surfaces, making them interesting for the design of developable strips. Indeed, if geodesics are known to be the shortest path between two points, another property (which is actually more general) is that the normal vector of a geodesic is the same as the normal vector of the surface at any point. This explains their "straightness property", which implies that when unrolled, developable strips cut along geodesics remain almost straight, minimizing the loss of material through cutting. This property is well known and used in the construction industry, especially for the cutting pattern of tensile structures [8].

\subsection{Discrete Monge's surfaces}

When both the generatrix and the rail curve are polylines, the resulting surfaces are naturally called discrete Monge's surfaces. Discrete Monge's surfaces are generated as depicted on Figure 3. Consider two consecutive edges on the rail curve $E_{i, j}$ and $E_{i, j+1}$. The next parallel of the discrete Monge's surface is generated with an offset of $E_{i, j}$ by a vector $\mathbf{V}$. The resulting line $E_{i+1, j}$ is intersected with $P$, the bissecting plane of $E_{i, j}$ and $E_{i, j+1}$, which give the new vertex of the parallel. The next edge $E_{i+1, j+1}$ is chosen to be parallel to $E_{i, j+1}$. This generation
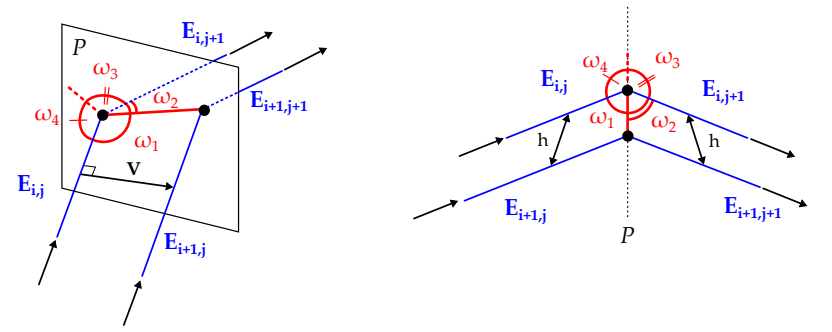

Figure 3: Generation of a discrete Monge surface by edge offset: perspective (left) and top view (right).

principle implies that there is a local symmetry for each node. It follows that the angles between the generatrix and two consecutive edges of a parallel are equal. These surfaces have therfore intrinsic properties that make them interesting for construction purpose:

- the panels are planar trapezoids. The parallelism of opposite edges makes the use of cost-effective standard opening mechanisms based on rack and pinions possible;

- the trapezoids have a constant height, which is optimal for nesting the panels within a rectangular bounding box;

- the nodes of discrete Monge's surface are torsionfree.
The first statements come directly from the generation principle presented on Figure 3. The last proposition can be proven by recalling that any conical mesh respects Equation (1), as proven in [22].

$$
\omega_{1}+\omega_{3}=\omega_{2}+\omega_{4}
$$

where $\omega_{i}$ are the angles between consecutive edges around a vertex. Here, due to symmetry with respect to the bissecting plane, one gets:

$$
\left\{\begin{array}{l}
\omega_{1}=\omega_{2} \\
\omega_{3}=\omega_{4}
\end{array}\right.
$$

which proves that discrete Monge's surfaces are indeed PQ-Conical Meshes.

The consequence of these propositions is that all Monge's surfaces can be considered as optimal for the most typical optimization goals. Indeed, they guarantee both faces planarity and torsion-free nodes. Nonetheless, the user has an intuitive understanding on how to generate them. This makes them interesting for typical applications of free-form structures such as glazed gridshells, an illustration of which is given in Figure 4. Some notorious examples of built moulding surfaces exist, like the SAGE Music Centre [4] or the Odate Dome [21]. This illustrates that moulding surfaces have entered the formal vocabulary of some architects. However, the property of conical mesh for Monge's surfaces did not seem to have been discussed before.

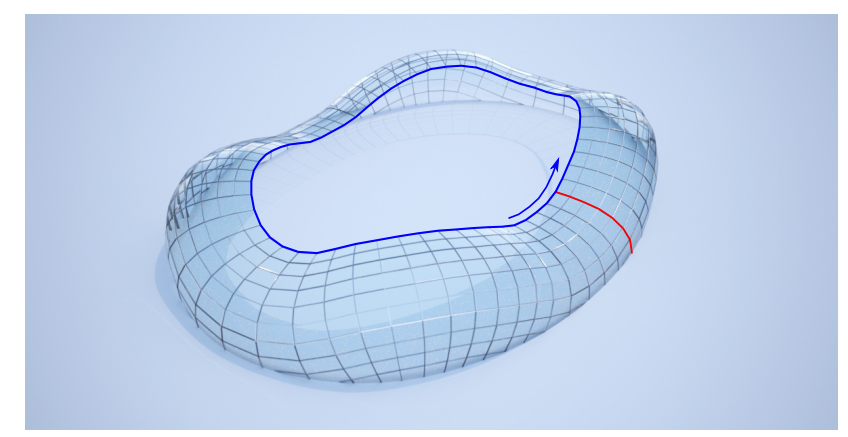

Figure 4: Free-form as a Monge's surface, the surface is covered with planar panels and torsion-free nodes.

\subsection{Moulding surfaces}

The concept of moulding surface was first introduced by Gaspard Monge, as a specific case of Monge's surface [12]. They were later studied by Gaston Darboux [5]. The design of moulding surfaces thus follows the simple procedure described in previous Section with a planar curve for the generatrix and a planar curve for the rail. Discretizing the surface following lines of curvature, it is noticed that the parameter influencing the angle at a vertex are limited. Indeed, as one can see on Figure 5, the only values of interest are the subdivision angle $\alpha_{i}$ of the rail (in blue) and 
the slope $\beta_{j}$ of the generatrix (in red) within the normal plane of the rail. A consequence is that for a given parallel of a discrete moulding surface, the angles of a vertex only depend on the subdivision angle of the rail. It follows that a moulding surface where the rail is a polyline with only one angle has a unique set of vertex angles for each parallel and therefore, that the nodes along the same parallel are identical.

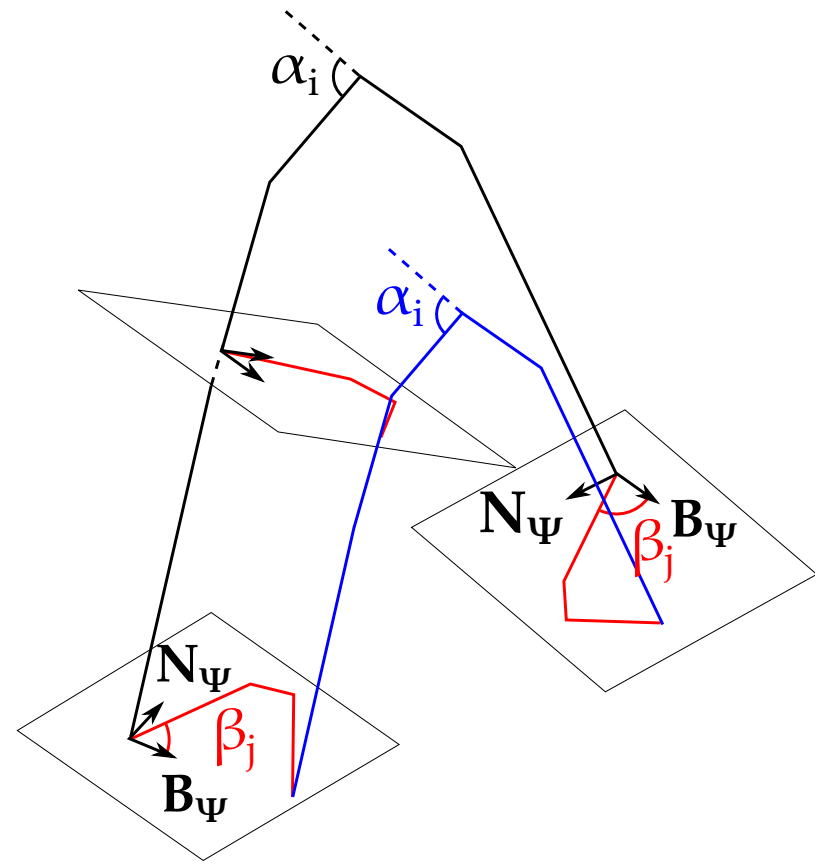

Figure 5: The angle of subdivision of the rail curve $\alpha_{i}$ and the slope of the generatrix with respect to the discrete rotation minimizing frame $\beta_{j}$ are the only parameters influencing the vertex angles.

\subsection{Isogonal moulding surfaces}

Consider a moulding surface where the rail curve is an isogonal line, i.e. a line with a constant angle subdivision. We call the resulting surface an isogonal moulding surface. Following statements can be made:

- for a given parallel of an isogonal moulding surface, all the vertices are identical;

- all the panels between two given consecutive parallels of an isogonal moulding surface are isosceles trapezoids;

- all the edges between two given consecutive parallels of an isogonal moulding surface are identical; The faces of isogonal moulding surfaces are inscribed in a circle, creating so-called circular meshes and the possibility to build with constant height nodes.

Isogonal moulding surfaces are therefore both circular and conical meshes. Indeed, isosceles trapezoids are the only trapezoids with a circumcircle and it has been seen in Section 3.2 that all discrete Monge's surfaces, and thus all discrete moulding surfaces are conical meshes.

The introduction of isogonal moulding surfaces gives therefore a simple way to compute quad meshes that are conical and circular meshes. They have a constant face or a constant vertex offset, giving interesting properties for fabrication. Therefore, the gain in cost of the connections for gridshells is made both by repeatability and by an intrinsic ease of manufacturing. Beyond the fact that a lot of nodes are identical, it seems that all nodes can be produced with the same rules, especially if the generatrix is an isogonal figure as well.

Although limited, the formal universe of isogonal moulding surfaces reveals archetypal shapes shapes in architecture, and some doubly-curved surfaces can easily be reinterpreted with this family of shapes. Figure 6 shows the rendering of a barrel vault interpreted as an isogonal moulding surface. The structure can be covered with planar quadrilateral panels and features remarkable offset properties. The rail curve is an parabolic arch with

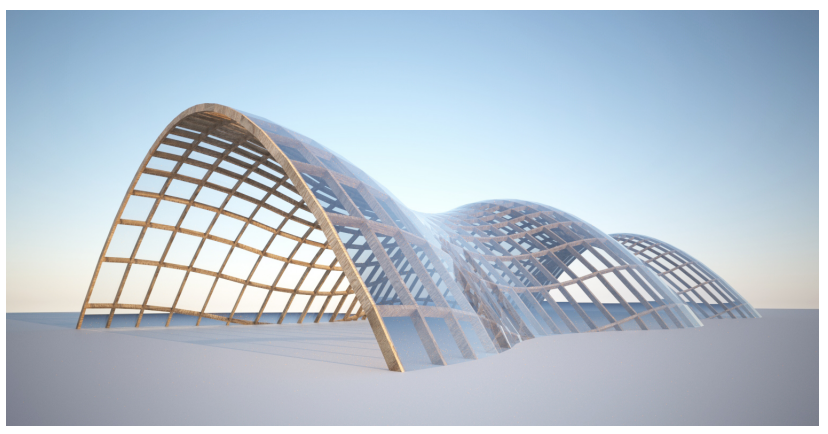

Figure 6: Free-form generated as an isogonal moulding surface.

20 nodes, there are 34 arches. Without geometrical rationalization, there would be 680 types of nodes. A regular isogonal moulding surface would have 34 types of nodes.

If there are $P$ peaks and $V$ valleys on the generatrix, then the number of different node types can be reduced with an appropriate isogonal subdivision of the generatrix. The number of different node types can be approached by Equation (3). The case of Figure 6 is illustrated on Figure 7: there are 2 peaks and 2 valleys, which reduces the total number of nodes to 8 .

$$
N_{\text {nodes }} \simeq \frac{N_{\text {parallels }}}{2(P+V)}
$$

Isogonal moulding surfaces can also be used to generate doubly-curved facades using a horizontal rail curve. The parallelism property implies that all the parallels (same family as the blue curve) are horizontal, simplifying the connection of the surface with an underlying structural layout. The generatrices (in red) can easily withstand loads such as self-weight, since they lay in a vertical plane and are likely to be main structural elements. The length 


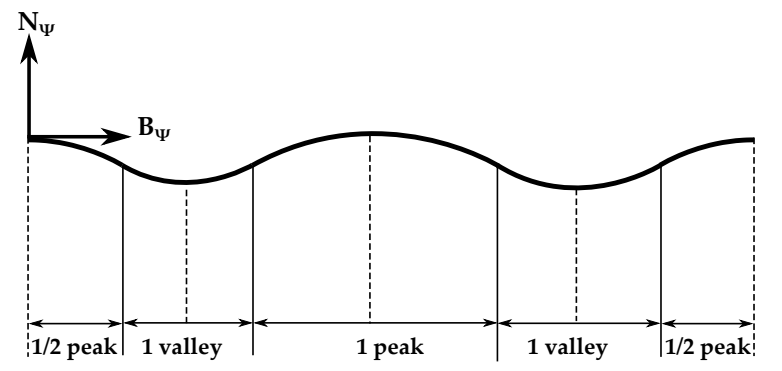

Figure 7: Peaks and valleys on the generatrix used on Figure 6

repetition property is here key to cost reduction: if the surface is an isogonal moulding surface, all the main structural elements will be identical. An example of application of these properties is the construction-aware design of stadia.

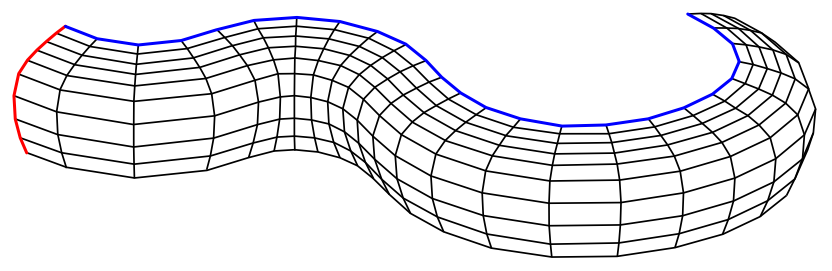

Figure 8: A vertical doubly-curved facade as an isogonal moulding surface, all the generatrices are equal.

\subsection{Mesh parallelism}

The remarkable properties of isogonal moulding surfaces can also be interpreted with elementary mesh parallelism. Figure 9 shows three parallel meshes. These meshes have the same combinatoric (same number of vertices, faces, edges and connectivity) and their respective edges are parallels [11]. It can be easily seen that isogonal
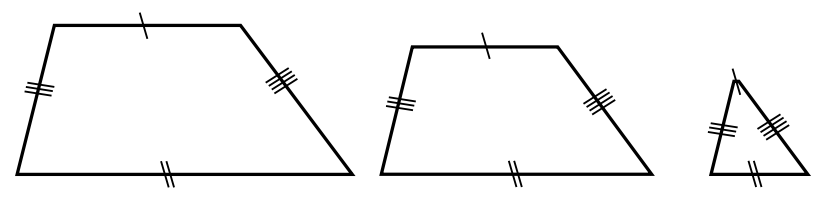

Figure 9: Illustration of mesh parallelism for a single planar polygon: all the figures are parallel to each other because their respective edges are parallel.

moulding surfaces are parallel to surfaces of revolution. Indeed, Figure 10 illustrates that a curve with no inflection point is parallel to a discrete curve on a circle. The application of this idea to the rail curve of an isogonal moulding surface gives the result. As a matter of fact, surfaces of revolution are particular cases of moulding surfaces where the rail-curve is a circle.

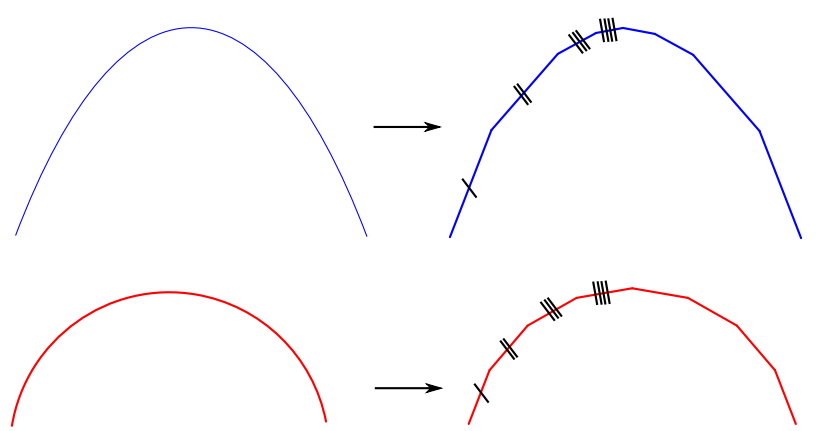

Figure 10: A spline and a portion of circle with the same integral of curvature. It is possible to compute parallel isogonal curves for these two curves.

The property of node repeatability is well-known for surfaces of revolution, provided that the parallels are isogonal curves: these meshes are called canonical meshes of surfaces of revolution. The repeatability of nodes in isogonal moulding surfaces can be explained with respect to this mesh-parallelism property. More importantly, isogonal moulding surfaces represent all the possible meshes parallel to the canonical mesh of surfaces of revolution. As particular cases of isogonal moulding surfaces, the faces of surfaces of revolution are trapezoids, and they feature a network of planar parallel curves. By definition, the property of parallelism is preserved on parallel meshes: all the parallel meshes of surfaces of revolution have trapezoidal faces and have a network of planar parallel curves. This is an alternative definition of moulding surfaces (see for example [12]), which proves that all the parallel meshes to surfaces of revolution are moulding surfaces. Mesh parallelism also preserves the angles, in particular, the angles of a parallel: all the possible meshes parallel to an isogonal moulding surface are therefore isogonal moulding surfaces.

This gives a very well-defined design framework with limited formal possibilities. However, the possible shapes of moulding surfaces are more diverse than the ones of surfaces of revolution. Figure 11 shows some isogonal curves that are parallel and will yield parallel meshes if associated to the same generatrix. The mesh generated on the left of the figure is a surface of revolution, whereas the others are isogonal moulding surfaces. These meshes can be built using identical nodes. The computational method presented in Section 4 makes the exploration of the formal possibilities of isogonal moulding surfaces intuitive and fully interactive. These tools have a practical interest for a formfinding purpose in a bottom-up approach. The numerical application also shows the trade-off between uniformity of vertices and uniformity in members lengths.

\subsection{Edge Offset Mesh and moulding surfaces}

Mesh parallelism can also be used to derive exact edge offset mesh from isogonal moulding surfaces. It has been shown in [15] that edge offset meshes feature vertices where 

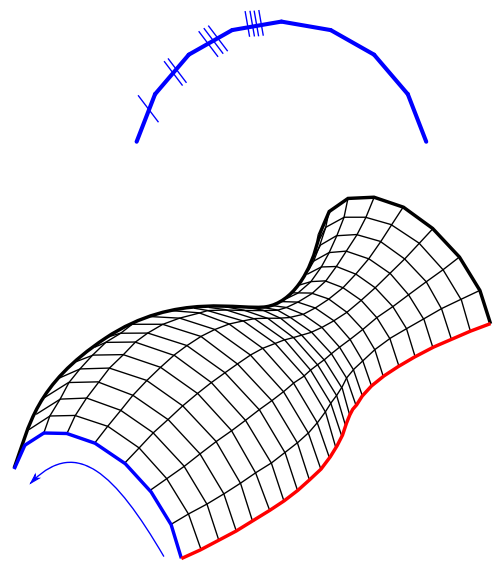
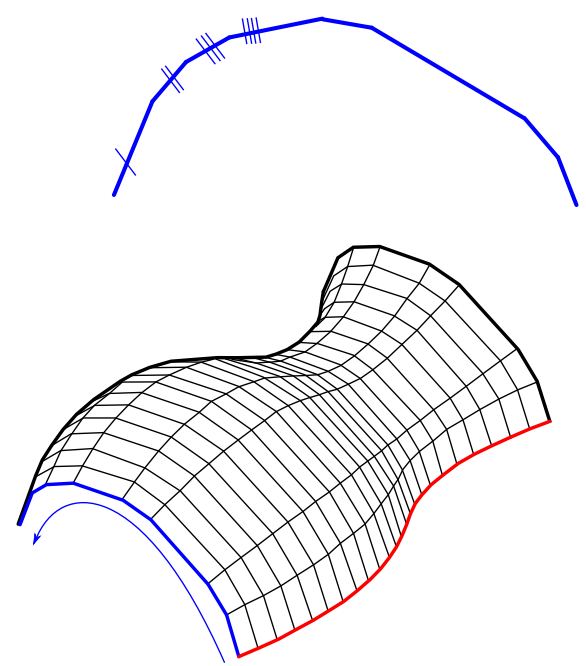

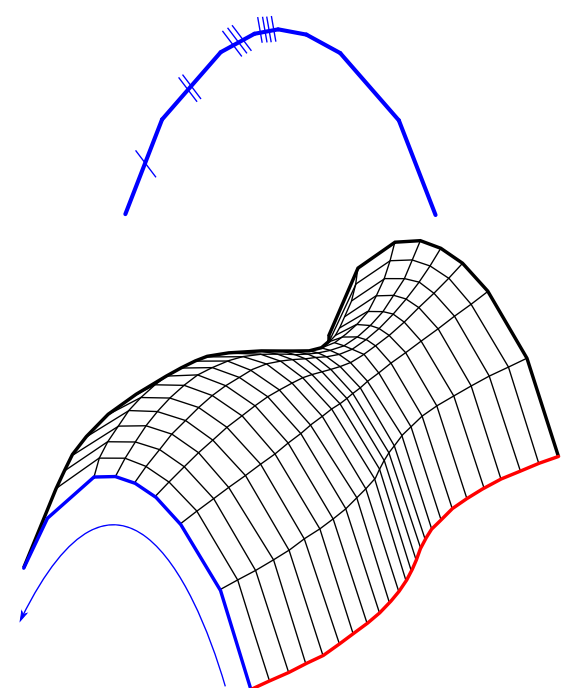

Figure 11: A set of parallel isogonal curves; if chosen as rail for moulding surfaces with a given generatrix, the resulting meshes will feature the same properties.

all edges are tangent to the same right circular cone. This makes them interesting from a technological perspective. They are parallel to meshes whose edges are tangent to a sphere known as Koebe meshes. The polyhedra of Figure 12 are Koebe meshes: their edges are tangent to a sphere, and, as a result, their faces have touching incircles. Many Koebe meshes exist, from Platonic solids such as the cube displayed on the left to more complex polyhedra.

As explained in Section 3.4, all the parallel meshes to meshes with a rotational symmetry are isogonal moulding surface. This means that all the possible shapes parallel to the canonical Koebe Meshes, such as the ones displayed on Figure 12 represent a subset of isogonal moulding surfaces. This section thus aims at explaining which moulding surfaces are constructible with edge offset mesh, and what kind of limitations this implies for a design purpose.
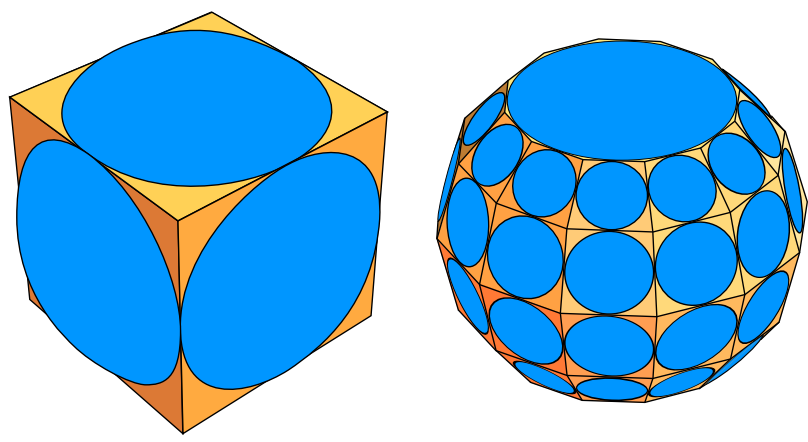

Figure 12: Canonical Koebe Meshes, the edges are tangent to a sphere and the incircles of the faces form a circle packing.

The construction of a Koebe mesh with a rotational symmetry is equivalent to a circle packing problem between two meridians of a sphere, as illustrated on Figure 13. Given $\Delta \theta$ an angular subdivision of the parallel, or equivalently two meridians (displayed in red) and $\lambda_{0}$, the latitude of the tangency point between an edge of the Koebe Mesh and the sphere (displayed in blue and dashed lines on the left of the Figure), the problem becomes onedirectional and can thus be solved very efficiently. The problem is indeed the construction of a circle that is tangent to the three aforementioned circles, which has two solutions (one circle on each side of the blue dashed circle). It is then possible to establish a non-linear recurrence for the admissible values of the latitude $\lambda$ defining tangency points between consecutive circles. Introducing $t_{i}=\tan \frac{\lambda_{i}}{2}$ and the constant $K_{\theta}=1+2 \sin ^{2} \frac{\Delta \theta}{2}$, the solution of this recurrence follows.

$$
t_{i+1}=\frac{2 t_{i} \pm\left(1-t_{i}^{2}\right) \sqrt{1-K^{2} \theta}}{\left(1-K_{\theta}\right) t_{i}+1+K_{\theta}}
$$

Recalling Figure 5 and the fact that the angles at a vertex of an isogonal moulding surface are only influenced by the slope of the generatrix in the normal plane of the rail, the recurrence relation leads to admissible values of $\lambda$, i.e. of admissible slopes for the subdivision of the generatrix. These values can be used on any isogonal moulding surface, leading to a propagation method which implementation is discussed in Section 4. The result is a method to generate edge offset meshes with a total control of the shape.

\section{Application and computational aspects}

\subsection{Isogonal subdivision of a convex planar curve}

The main challenge left with the design of isogonal moulding surfaces is the isogonal subdivision of a planar curve. A strategy that guarantees an isogonal subdivision of a smooth convex curve is proposed in this section. The method can be interpreted graphically, as pictured in Figure 14. The basic algorithm steps follow: 

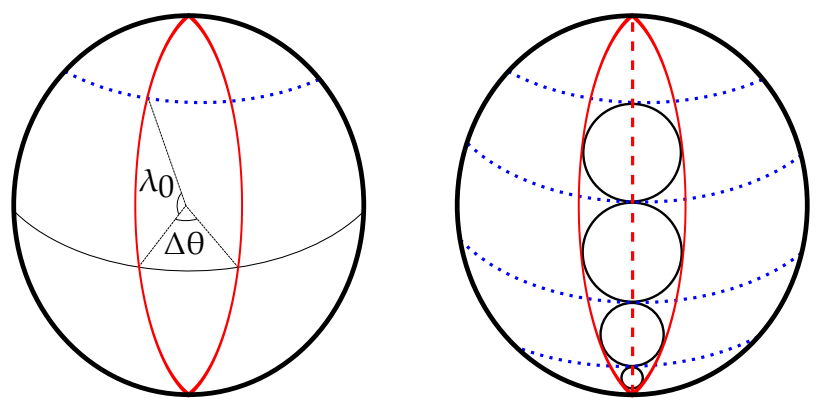

Figure 13: Perspective view of the parameters of the circle packing problem: for two given meridians (continuous) and one parallel (dotted), a unique solution exists.

1. Find the tangent vectors $\mathbf{T}_{\mathbf{A}}$ and $\mathbf{T}_{\mathbf{B}}$ at the ends of the curve, and measure their angle $\alpha_{A B}$ (if the curve is closed and convex, chose $\alpha_{A B}=2 \pi$ );

2. Divide $\alpha_{A B}$ by the desired number $n$ of inner nodes. Create the vectors $\left(\mathbf{T}_{\mathbf{i}}\right)_{i=1}^{n-1}$, where each $\mathbf{T}_{\mathbf{i}}$ is obtained by a rotation of $\mathbf{T}_{\mathbf{A}}$ by an angle of $\alpha_{A B} \cdot i / n$

3. Find the points corresponding to the tangency to the $\left(\mathbf{T}_{\mathbf{i}}\right)_{i=1}^{n-1}$ on the initial curve;

4. Intersect the corresponding lines with each other.
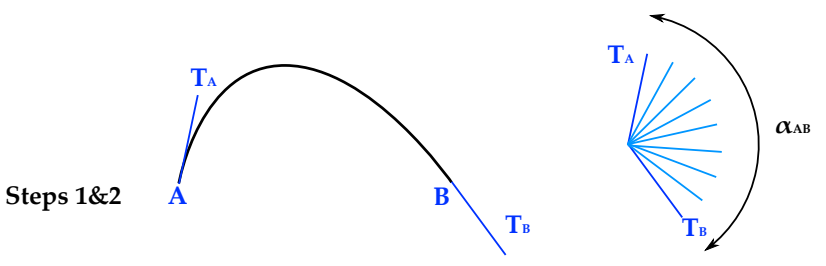

Step 3
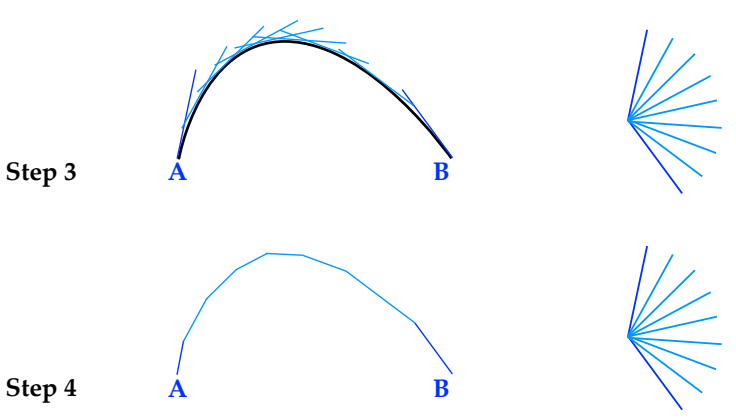

Figure 14: A graphical method for the isogonal subdivision of a planar curve.

With this formulation, only angles that are a unit fraction of the total angular variation of the tangent vector are admissible.

$$
\alpha_{e x t}=\frac{\alpha_{A B}}{n}
$$

It is possible to be even more specific: the points of tangency $P_{i}$ found at step 3 form a partition of the curve with a constant integral of curvature.

$$
\alpha_{e x t}=\left(\mathbf{T}_{\mathbf{i}}, \mathbf{T}_{\mathbf{i}+\mathbf{1}}\right)=\int_{P_{i}}^{P_{i+1}} \kappa d s
$$

Intuitively, the consequence is that areas with low curvature will feature longer elements, which can already be noticed on Figures 6 and 14. This criterion also means that fully numerical approaches based on the integral of curvature are applicable to the search of isogonal polylines.

It has to be noticed that the algorithm can be made more flexible by letting the user prescribe an angle of subdivision and a first point of tangency. The last two steps of the method described above can be implemented with the prescribed value of the subdivision angle.

\subsection{Isogonal subdivision of a planar curve}

Consider now the case of a curve that is not convex and more precisely with varying curvature signs. The strategy proposed above does not apply directly, but it can be generalised. Indeed, the procedure to follow is simple:

1. Find the inflection points of the curve and use them to make a partition of the curve ;

2. Apply the procedure described in Section 4.1 for each part.

By doing so, one is sure that each part will have an isogonal subdivision. The last problem to solve is to know whether it is possible or not to have the same subdivision for each part with the simple graphical method described above. Equation (6) gives an answer to this problem: it is possible to have the same subdivision angle for two parts whose integral of curvature are commensurable (their ratio must be a rational number). This restriction is too strong for a general purpose. For this reason, a propagation algorithm where the user chooses the angle of subdivision is more convenient. The algorithm implemented follows the same principle of tangency point and adapts the sign of the subdivision angle by checking the sign of the curvature.

The algorithm proposed hereinabove has been implemented as a PythonScript in the drawing software Rhinoceros. The benchmark for the isogonal subdivision algorithm studies two typical curves. The first one is a parabola and has no inflection point. The second one is a fourth order spline with four inner inflection points recalling a sine function. Both curves are shown on Figure 15. The inflection points and tangency points are found by means of a binary search algorithm.

As seen on Table 2, the computation time is low, even for a large number of subdivisions. It can be noticed that the convergence of the algorithm does not vary linearly with the number of inner nodes: doubling the number of inner nodes will not double the computation time. The partition into several domains with the inflection points makes the computation slower for the sine curve. These examples show that the algorithm proposed allows a realtime manipulation of isogonal planar curves and isogonal moulding surfaces.

\subsection{Computation of edge offset meshes from moulding surface}

The computation of edge offset meshes from isogonal moulding surfaces is closely linked to the construction of a 


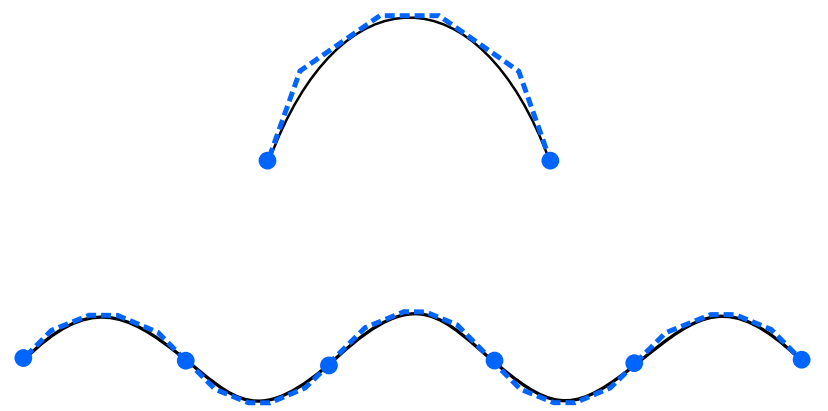

Figure 15: Benchmark curves (continuous) with end points and inflection points, and the result of our algorithm (dashed lines).

\begin{tabular}{|c|c|c|}
\hline $\begin{array}{c}\text { Type } \\
\text { of curve }\end{array}$ & Number of inner nodes & $\begin{array}{c}\text { Computation } \\
\text { time }[\mathrm{s}]\end{array}$ \\
\hline & 25 & 0.04 \\
Parabola & 50 & 0.06 \\
& 100 & 0.12 \\
& 200 & 0.21 \\
\hline \multirow{3}{*}{ Pseudo-Sine } & 25 & 0.10 \\
& 50 & 0.11 \\
& 100 & 0.17 \\
& 200 & 0.27 \\
\hline
\end{tabular}

Table 2: Computation time for the isogonal subdivision algorithm, the computation was performed on a computer with $2.4 \mathrm{GHz}$ and 2 GB memory.

Koebe mesh with a rotational symmetry, see Section 3.6. Such a mesh can be determined with two given meridians on a sphere, or equivalently, with a subdivision angle of the parallels, as a starting point. Due to its symmetries, the problem to solve can be represented on a plane, as shown in Figure 16. Consider the bisecting plane of the two meridians. The projection of these curves into the plane gives an ellipse (dashed figure), whereas the meridian in the bisecting plane is a circle (thick continuous line). The Koebe Mesh has its edges tangent to the sphere, which means that the projection of an edge on a meridian (blue continuous line) has to be tangent to the ellipse. In the plane displayed on Figure 16, the projection of an edge along a parallel is a point (in blue on the figure). The condition of edge tangency for Koebe mesh means that this point has to be on the central meridian (thick black circle). The symmetry of the problem guarantees indeed that the contact has to be on the central meridian and on the two pre-determined meridians. By this mean, starting from a point $P_{1}$, one is able to find a point $P_{2}$ so that the line $\left(P_{1} P_{2}\right)$ is tangent to the ellipse at the point $P_{1}^{\prime}$. The procedure can be repeated to construct other points $P_{2}^{\prime}$ and $P_{3}$, and so on.

For a given point $P_{1}$ and a propagation direction (either towards the North pole $N$ or the South pole $S$ ), there is one unique way to construct a valid trapezoid that respect the rotational symmetry and the edge offset property. This

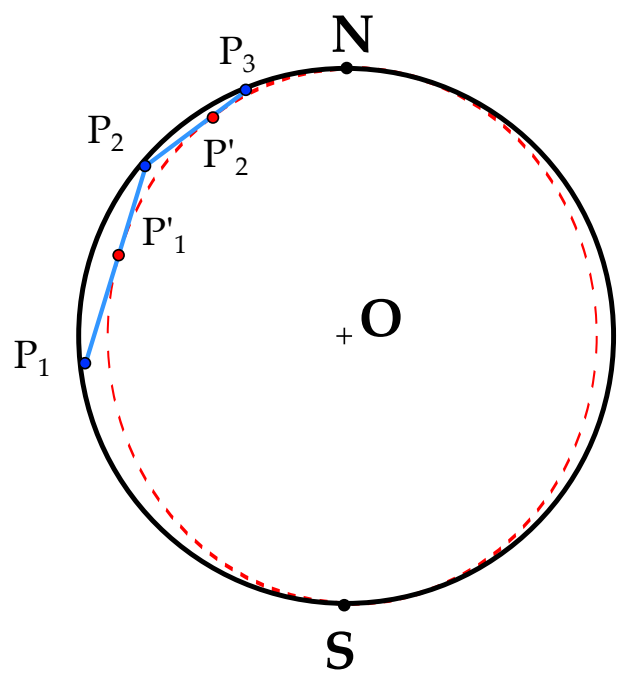

Figure 16: Propagation technique for a sphere, the dashed line is the projection of a meridian within the plane of the central meridian

leads to the incremental construction of the sets of points $\left(P_{i}\right)_{i=1}^{n}$ on the circle and $\left(P_{i}^{\prime}\right)_{i=1}^{n-1}$ on the ellipse. The key information for the construction of edge offset mesh on moulding surface is the computation of the slope of the generatrix, which is the slope of the line $\left(P_{i-1} P_{i}\right)$. This calculation gives therefore admissible values of the slope of the generatrix for the construction of an edge offset mesh. The computation is very efficient, since the problem is fundamentally one-directional.

Once the problem of the Koebe mesh on the sphere is solved, it is possible to apply this approach to any moulding surface. The procedure follows:

1. Subdivide the rail curve into an isogonal figure;

2. Measure the initial slope of the generatrix;

3. Determine the propagation direction (North pole or South pole);

4. Solve the equivalent problem on the sphere with these initial values (the result is a set of tangent vectors);

5. Find the tangency points on the generatrix, a suitable curve for an edge offset mesh being the envelope of the tangent lines.

The simplicity of this formulation leads to good computational performances, the calculation time being typically below one second, even for meshes with thousands of faces. A set of typical values of computation is given in Table 3, the geometry considered is a mesh on a sphere with a symmetry with respect to the equator, as the mesh displayed on Figure 12. In this case, the computation time is mainly governed by the isogonal subdivision of the rail curve.

The method presented hereinabove is not free from simplifications and from hypothesis. The first one is that the generatrix has to exhibit curvature in order to use the propagation technique. If it is not the case, the algorithm 


\begin{tabular}{|c|c|c|c|}
\hline$\Delta \theta$ & $\Delta \lambda$ & Number of Faces & $\begin{array}{c}\text { Computation } \\
\text { time }[\mathrm{s}]\end{array}$ \\
\hline \multirow{5}{*}{$5^{\circ}$} & $30^{\circ}$ & 504 & 0.19 \\
& $60^{\circ}$ & 936 & 0.20 \\
& $120^{\circ}$ & 2232 & 0.24 \\
& $160^{\circ}$ & 3384 & 0.32 \\
\hline \multirow{5}{*}{$15^{\circ}$} & $30^{\circ}$ & 72 & 0.18 \\
& $60^{\circ}$ & 120 & 0.20 \\
& $120^{\circ}$ & 264 & 0.22 \\
& $160^{\circ}$ & 384 & 0.16 \\
\hline
\end{tabular}

Table 3: Computation time for the Edge Offset Mesh algorithm for a sphere.

will fail to find admissible values for the slope of the generatrix. This is rather an intrinsic limitation of these surfaces than a weakness of the algorithm. For example, a cylinder cannot be given an edge offset mesh, since its Gaussian map is a circle.

Another difficulty in the use of the edge offset mesh algorithm is that the procedure described does not allow to cover the entire sphere with a quad mesh. Graphically, it can be seen on Figure 16 that the ellipse and the circle get closer as one moves away from the equator, which means that each "step" is smaller. Figure 12 shows this narrowing of the faces as the latitude is larger. Numerically, this means that the algorithm, as it is presented here, would not converge if the normal of the generatrix is the same as the normal of the plane containing the rail curve, which corresponds to the poles in the case of a sphere. From a practical point a view, it is therefore necessary to prescribe a minimal angle between these normals to stop the computation. An illustration of the practical limitations of Koebe mesh on moulding surfaces with respect to this point is given on Figure 17, where a torus is meshed with our algorithm. The narrowing of the elements towards the top of the small circle is clearly visible, and would be likely to be considered undesired from an architectural and technological point of view. The mesh exhibited is still an edge offset mesh, but it does not only feature quad elements. This simple shape illustrates some of the intrinsic limitation of edge offset meshes in practical cases.

\subsection{Gridshells with planar faces}

In this section, an evaluation of the design possibilities offered by moulding surfaces, isogonal moulding surfaces and edge offset meshes for gridshells with planar faces is proposed. In particular, the analytical link between the discrete mesh and the smooth surfaces leads to some remarks on the malleability of edge offset meshes.A case study is proposed for two archetypical examples of doubly curved shapes in architecture: domes and barrel vaults.

Moulding surfaces are indeed well-suited for the generation of domes. The form generation principle is illustrated on Figure 18: the rail curve is vertical and the generatrix

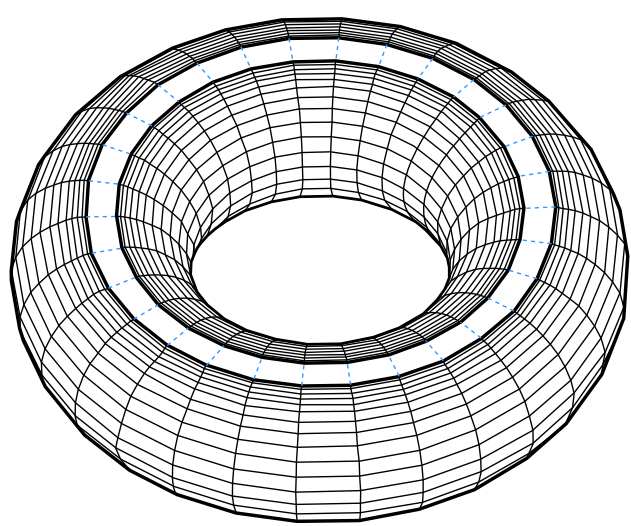

Figure 17: Edge offset mesh on a torus, a particular case of isogonal moulding surface where the algorithm proposed in this article does not provide a mesh with quads only due to intrinsic limitations of the shape.

is a curve with no inflection point. The surface generated is cut by an horizontal plane in order to illustrate possible real life application. Three geometries of dome are considered: the rail curve (in blue) is a third order spline and remains unchanged, whereas the generatrix (in red) varies. This gives three geometries: an oblong shape, a nearly-spherical one and an intermediate shape. A relatively coarse and a fine edge offset mesh have been generated on each shape, the coarse meshes can be seen on Figure 19. The computation of the meshes is instantaneous.
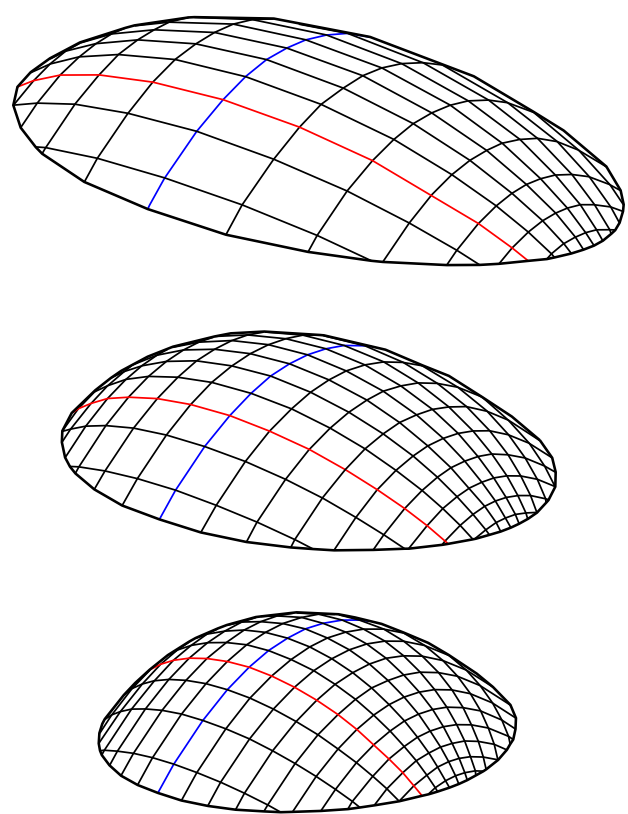

Figure 19: Perspective view of the three domes as edge offset meshes.

The number of subdivisions of the rail-curve is identical for the three geometries, which implies that all the three edge offset meshes are parallel to each other and derive from the same Koebe mesh on the sphere. The aspect 

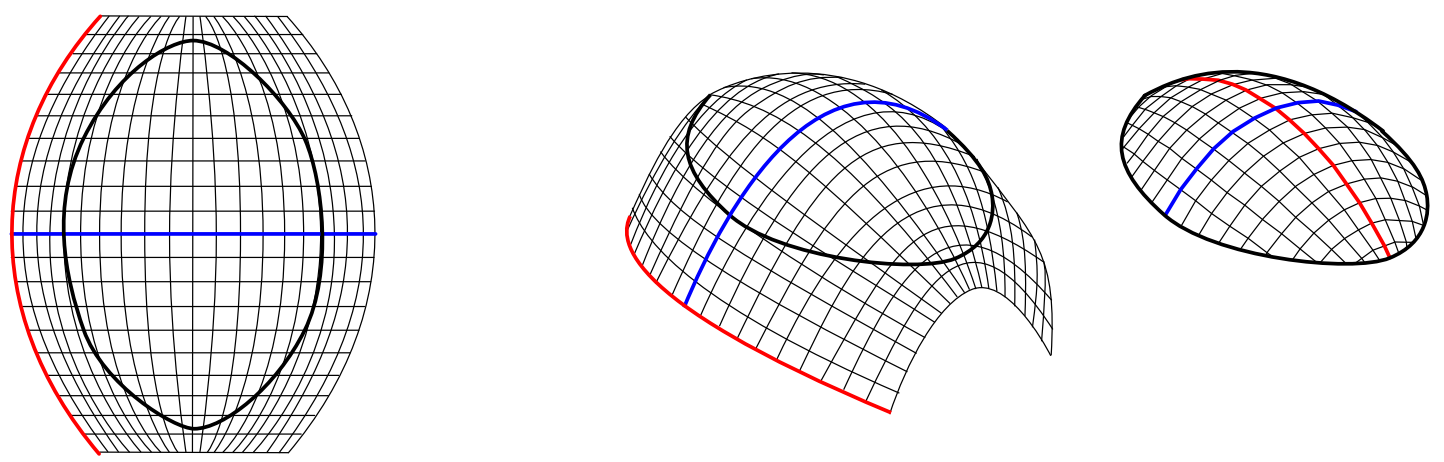

Figure 18: Dome as a moulding surface: top view and isometric view.

ratio of a panel is defined as the ratio of its length (maximum of the mean value of the lengths of two opposite edges) over its width (minimum of the mean value of the lengths of two opposite edges). In Table 4, a comparison between the aspect ratio of the panels $L / l$ and the ratio of principal curvatures at the apex of the dome $R_{1} / R_{2}$ is made. It appears that these quantities are very similar, especially when the mesh is refined. For 50 subdivisions of the rail curve, the difference between these quantities is below $2 \%$. This simple example shows that the ratio of principal curvatures is likely to be a key factor in the anisotropy of edge offset meshes. Keeping this in mind, some precautions can be taken to improve the quality of an edge offset mesh, or controlling intuitively its anisotropy:

- Reduce the variation of curvature of the rail curve;

- Avoid areas with low gaussian curvature, as they are hard to cover with edge offset meshes;

- Even the curvature between parallel and generatrix in order to deal with eventual mesh anisotropy.

\begin{tabular}{|c|c|c|c|}
\hline $\begin{array}{c}\text { Number of } \\
\text { subdivisions of } \\
\text { the rail curve }\end{array}$ & Type of Dome & $L / l$ & $R_{1} / R_{2}$ \\
\hline \multirow{2}{*}{15} & Oblong & 3.50 & 3.60 \\
& Intermediate & 1.88 & 2.01 \\
& Quasi-spherical & 1.12 & 1.13 \\
\hline \multirow{2}{*}{50} & Oblong & 3.53 & 3.60 \\
& Intermediate & 2.02 & 2.01 \\
& Quasi-spherical & 1.13 & 1.13 \\
\hline
\end{tabular}

Table 4: Relation between aspect ratio of the panels $L / l$ and the ratio of the curvatures $R_{1} / R_{2}$.

Isogonal moulding surfaces are also of interest for the generation of shapes with varying curvature, such as corrugated barrel vaults. Figure 20 demonstrates this possibility: the rail curve (in blue) is a B-spline of degree 3 with a rise over span ratio of $37.5 \%$. The generatrix is a sine curve which is also divided as an isogonal polyline (with the exception of the inflection points, where flat node are introduced). The structure features nearly 700 nodes, but thanks to the isogonal moulding surface properties and to the symmetries of the shape, only 8 types of nodes are used. Height lengths of members are required to span between any parallels.

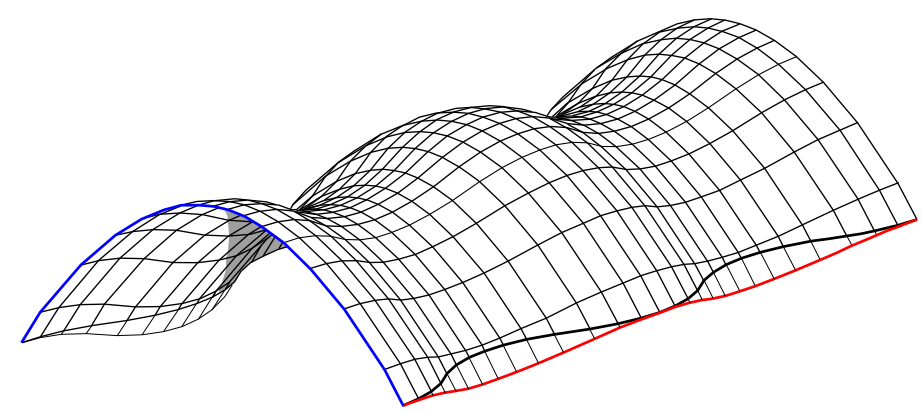

Figure 20: Corrugated barrel vault as an isogonal moulding surface.

It can be noticed on Figure 20 that the panels are longer towards the supports of the structure, which can be explained by equation (6): the isogonal subdivision has to define a constant integral of curvature for the rail curve. The Figure 21 shows that the curvature is bigger on the top of the rail curve, which is a well known fact for curves such as parabolæ for example. The dots represent the points of tangency found with our algorithm. This phenomenon of shortening of elements in areas of high curvature can be problematic if the variations are important. In this case, the designer can choose to divide straight lines by adding intermediary elements or to divide the rail curve with two angles, instead of only one.

\subsection{Form-finding procedure}

The shape of Monge's surface depends on both the rail curve and generatrix. In order to modify a design, one can therefore use different options, that are similar to those dictating the design of scale-trans surfaces [7]:

- modify the control points of the rail curve; 


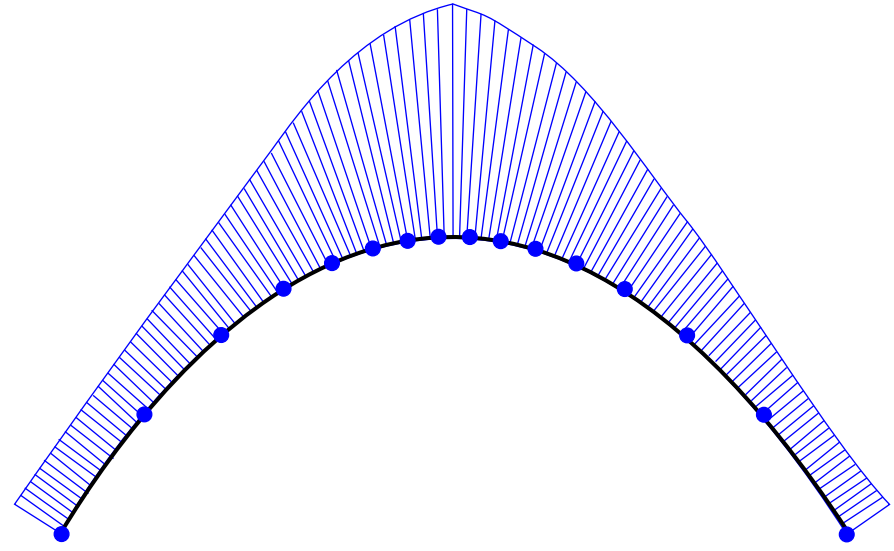

Figure 21: Curvature graph of the rail curve in Figure 20, the dots represent the tangency points found with the algorithm presented in this paper.

- modify the control points of the generatrix;

- adjust the position of the generatrix within the normal plane of the rail curve.

The different offset properties of isogonal moulding surfaces make it easy to generate mesh offset: the structural engineers can work with the mid-lines of the beams, whereas offsets can be used to produce shop drawings of glass panels. The process can be fully automated with limited scripting effort.

\section{Conclusion}

The use of parametric tools allows the design of complex shapes. The development of NURBS modeling has triggered a real transformation of the industry. The exploration of the typical construction constraints is now made easier with methods working on general shapes. However, the limit between what could be called a "simple" freeform and a complex one remains blurred and the question of surfaces that are inherently good from a geometrical prospective is still an open question. With this work, a family of intrinsically simple shapes that the designers can easily and intuitively manipulate was proposed, as shown in Section 4. Monge's surfaces and their discrete counterpart are indeed a rich family of PQ conical meshes that can be generated very easily in modeling software applications.

It was shown that circular meshes and edge-offset meshes of moulding surfaces require a subdivision of the rail curve with a constant integral of curvature, which is an original illustration of the trade-off between length uniformity and vertex uniformity in doubly curved structures. Isogonal moulding surfaces also represent all the possible meshes parallel to canonical meshes on surfaces of revolution.

The authors also consider isogonal moulding surfaces as the first example of surfaces where high node congru- ence can be achieved, opening new possibilities for manufacturing. The performance of these forms is illustrated in Table 5. In this sense, they can be compared to translation surfaces, which triggered reflections on face planarity and led to powerful geometrical procedures. Both types of surfaces suffer from the same limitations regarding the formal freedom left to the designers: not every shape can be described as a moulding surface (example: an ellipsoid), but archetypal shapes such as domes, stadia or barrel vaults could be reinterpreted easily with this family of meshes.

The remarkable properties of isogonal moulding surfaces and of Monge's surfaces assure an easy manufacturing of all kinds of structural systems, from construction of formwork with planks for concrete shells to steel gridshells or even double layer grid structures. The great amount of combined properties and the interactivity of the tools shown in this paper allow the designers to explore various structural systems at early stages of design while keeping the same network of discrete lines. These structural possibilities should be investigated in the future. The bottom-up strategy described in this article is complementary with more general approaches such as PQ meshes computed from curvature lines. Both strategies will lead to different projects and shapes, but they are both valid and interesting.

\section{Aknowledgement}

An aknowledgement should be made to Bouygues Construction and the ANRT, who founded this research. The authors would also like to thank Yann Santerre for the nice renderings.

\section{References}

[1] Bayerel, O., Caron, J.-F., Tayeb, F., and Peloux, L. Gridshells in composite materials: Construction of a $300 \mathrm{~m}^{2}$ forum for the solidays' festival in paris. Structural Engineering International: Journal of the International Association for Bridge and Structural Engineering (IABSE) 22, 3 (2012), 408-414.

[2] Blassel, J., And Pfadler, A. La gare de strasbourg. Construction métallique 45, 1 (2008), 15-36.

[3] Bouaziz, S., Deuss, M., Schwartzburg, Y., Weise, T., And Pauly, M. Shape-Up: Shaping Discrete Geometry with Projections. Computer Graphics Forum 31, 5 (Aug. 2012), 1657-1667.

[4] Cook, M., Palmer, A., And Sischka, J. Sage music centre, gateshead-design and construction of the roof structure. Structural Engineer 84, 10 (2006), 23.

[5] Darboux, G. Leçons sur la théorie générale des surfaces et les applications géométriques du calcul infinitésimal. 1915.

[6] Douthe, C., Caron, J., And Baverel, O. Gridshell structures in glass fibre reinforced polymers. Construction and Building Materials 24, 9 (Sept. 2010), 1580-1589.

[7] Glymph, J., Shelden, D., Ceccato, C., Mussel, J., And SCHOBER, H. A parametric strategy for free-form glass structures using quadrilateral planar facets. Automation in Construction 13, 2 (Mar. 2004), 187-202.

[8] Gründig, L., Ekert, L., And Moncrieff, E. Geodesic and Semi-Geodesic Line Algorithms for Cutting Pattern Generation of Architectural Textile Structures. Proceedings of the AsiaPacific Conference on Shell and Spatial Structures, Beijing. (1996). 


\begin{tabular}{|c|c|c|c|c|c|}
\hline Optimisation & $\begin{array}{c}\text { Angles } \\
\text { repetition }\end{array}$ & $\begin{array}{c}\text { Length } \\
\text { uniformity }\end{array}$ & $\begin{array}{c}\text { Panels } \\
\text { planarity }\end{array}$ & $\begin{array}{c}\text { Node } \\
\text { complexity }\end{array}$ & $\begin{array}{c}\text { Design } \\
\text { freedom }\end{array}$ \\
\hline Triangular Mesh & - & ++ & ++ & -- & ++ \\
\hline Scale-trans surfaces & -- & ++ & ++ & - & -- \\
\hline PQ Conical Mesh & + & + & ++ & + & + \\
\hline Edge Offset Mesh & + & - & ++ & ++ & - \\
\hline Isogonal moulding surfaces & ++ & + & ++ & ++ & -- \\
\hline
\end{tabular}

Table 5: Isogonal moulding surfaces compared to general optimisation methods.

[9] Happold, E., And Liddell, W. Timber lattice roof for the Mannheim Bundesgartenschau. The structural engineer 53, 3 (1975), 99-135.

[10] Knippers, J., And Helbig, T. Recent Developments in the Design of Glazed Grid Shells. International Journal of Space Structures 24, 5 (2009), 111-126.

[11] Liu, Y.and Wang, W., Pottmann, H., Wallner, J., And Yong-LiAng, Y. Geometric Modeling with Conical Meshes and Developable Surfaces. ACM Trans. Graphics 25, 3 (2006), $681-689$

[12] Monge, G. Application de l'analyse à la géométrie, à l'usage de l'Ecole impériale polytechnique. 1807.

[13] Отто, F. IL10 Gitterschalen. Institut für leichte Flächentragwerke (IL), Stuttgart, 1974.

[14] Pottmann, H., Grohs, P., And Blachitz, B. Edge offset meshes in Laguerre geometry. Advances in Computational Mathematics 33 (2010), 45-73.

[15] Pottmann, H., Liu, Y., Wallner, J., Bobenko, A., And WANG, W. Geometry of multi-layer freeform structures for architecture. ACM Transactions on Graphics 26, 3 (July 2007), 65.

[16] Pottmann, H., Schiftner, A., Bo, P., Schmiedhofer, H., Wang, W., Baldassini, N., And Wallner, J. Freeform surfaces from single curved panels. ACM Transactions on Graphics 27, 3 (Aug. 2008), 1.

[17] Schlaich, J.And Schober, H. Glass Roof for the Hippo Zoo at Berlin. Structural Engineering International 7, 4 (1997), 252254.

[18] Schlaich, J., And Bergermann, R. Leicht Weit: Jörg Schlaich-Rudolf Bergermann. Prestel, 2003.

[19] Schlaich, M., Burkhardt, U., Irisarri, L., and Goñi, J. Palacio de comunicaciones-a single layer glass grid shell over the courtyard of the future town hall of madrid. In Symposium of the International Association for Shell and Spatial Structures (50th. 2009. Valencia). (2010).

[20] Stephan, S., Knebel, K., and Sanchez-Alvarez, J. Reticulated Structures On Free-Form Surfaces. Stahlbau 73 (2004), $562-572$.

[21] Tanno, Y., Kozuka, H., And Nakai, M. Odate dome, japan. In IASS Symposium 2001: International Symposium on Theory, Design and Realization of Shell and Spatial Structures, Nagoya, Japan, 9-13 Oct. 2001 (2001), pp. 372-373.

[22] Wang, W., Wallner, J., And LiU, Y. An angle criterion for conical mesh vertices. Geometry Pre-Print 157 (2006), 1-10.

[23] Yang, Y.-L., Yang, Y.-J.. Pottmann, H., and Mitra, N. J. Shape Space Exploration of Constrained Meshes. ACM Trans. Graphics 30 (2011), 124. 